\title{
Microwave induced forward scattering and Luttinger liquid interferences in magnetically confined quantum wires
}

\author{
Alain Nogaret \\ Department of Physics, University of Bath, Bath BA2 7AY, UK \\ E-mail: A.R.Nogaret@bath.ac.uk \\ Jean-Claude Portal \\ High Magnetic Field Laboratory, 25 Avenue des Martyrs, 38042 Grenoble, France \\ Institut Universitaire de France and INSA, 31077 Toulouse, France \\ Harvey E. Beere and David A. Ritchie \\ Cavendish Laboratory, University of Cambridge, CB3 OHE Cambridge, UK \\ Chris Phillips \\ Department of Physics, Imperial College, London SW7 2AZ, UK \\ Received March 31, 2008
}

\begin{abstract}
We report on the photoresistance of a magnetic quantum wire obtained by applying a gradient of magnetic field to a two-dimensional electron gas. Electron transmission through the magnetic wire increases by an order of magnitude under microwave irradiation and exhibits frequency dependent magneto-oscillations as a function of the in plane magnetic field. Both results are fully consistent with microwave coupled Luttinger liquid edge channels which interfere at two pinning sites in the fashion of a Mach-Zehnder interferometer.
\end{abstract}

PACS: 73.21.Hb Quantum wires.

Keywords: Luttinger liquid, magnetically confined quantum wires, correlated electrons, microwaves.

In one dimension, the electron gas condenses into a chain of strongly interacting particles called the Luttinger liquid (LL) [1]. This liquid supports charge density wave instabilities which are totally reflected even by the weakest link in the chain [2-4]. The recent observation of power law behavior characteristic of the LL insulator, in the thermoactivated [5-7] and tunnelling current [8,9], appears as a fundamental obstacle to scaling down electronic devices to the size of single mode metal wires [10-14]. Here, however, we report the first observation of quantum interferences in a multi-channel LL $[15,16]$ coupled by a microwave field at two pinning sites where electrons experience forward scattering. Experimental data fit quantitatively the response of the LL confined by a spatially varying magnetic field $[17,18]$ and yield the distance between pinning centres $(11 \mu \mathrm{m})$ as the only adjustment parameter. The enhancement of quantum coherence by Coulomb interactions elicits applications to $\mathrm{THz}$ detectors [19], quantum computation $[3,17,20,21]$ and provides new insight into correlated electron systems [9,22-24].

Magnetically confined quantum wires (MCQW) were obtained by fabricating dysprosium micro-strips (length $40 \mu \mathrm{m}$; width $200 \mathrm{~nm}$; height $150 \mathrm{~nm}$ ) at the centre of narrow Hall bars (width $1.6 \mu \mathrm{m}$; length $32 \mu \mathrm{m}$ ) made of a GaAs/AlGaAs quantum well (density $n_{s}=4.0 \cdot 10^{11} \mathrm{~cm}^{-2}$, mobility $m=$ $=0.31 \cdot 10^{6} \mathrm{~cm}^{2} /(\mathrm{V} \cdot \mathrm{s})$ set $30 \mathrm{~nm}$ from the surface [25]. A magnetic field $B$ was applied in the plane to magnetize the stripe perpendicular to its long axis as shown in Fig. 1 (inset). This had the effect of exposing the two-dimensional electron gas (2DEG) to a fringing magnetic field $B_{m}(x)$ of amplitude varying between 0 and \pm 0.9 T. The magnetic field $B$ was aligned in the plane to a high accuracy $\left(<0.8^{\circ}\right)$ and had no direct effect on the 2DEG. An optically transparent titanium film (30 nm thick) capped the magnetic wire to screen re- 
sidual electrostatic potentials and to protect the dysprosium from oxidation. Samples were cooled to $1.3 \mathrm{~K}$. Microwaves were applied via an over-moded circular waveguide terminated by linear polarizers and a $45^{\circ}$ mirror redirecting power onto the sample $(\mathbf{E} \perp$ wire). The output power was varied from 0 to $20 \mathrm{~mW}$ using a variable attenuator. The photoresistance was measured under quasi-dc conditions using a small current excitation (100 nA) which gave a spectral width $(\sim 0.1 \mathrm{mV})$ comparable to thermal broadening.

Figure 1 shows the magnetoresistance of a $24 \mu \mathrm{m}$ long MCQW with and without microwave irradiation. This exhibits a drop from 7.1 to $0.6 \mathrm{k} \Omega$ when microwave power increases from 0 to $20 \mathrm{~mW}$. In comparison, the resistance of the bare Hall bar is $0.18 \mathrm{k} \Omega$ which is consistent with the resistance of a channel of nominal width supporting 75 modes. A much smaller photo-resistance was observed across shorter channel lengths with typically $\Delta R / R=-2 \%$ across a $4 \mu \mathrm{m}$ segment. Surprisingly, microwaves induce complex magneto-resistance

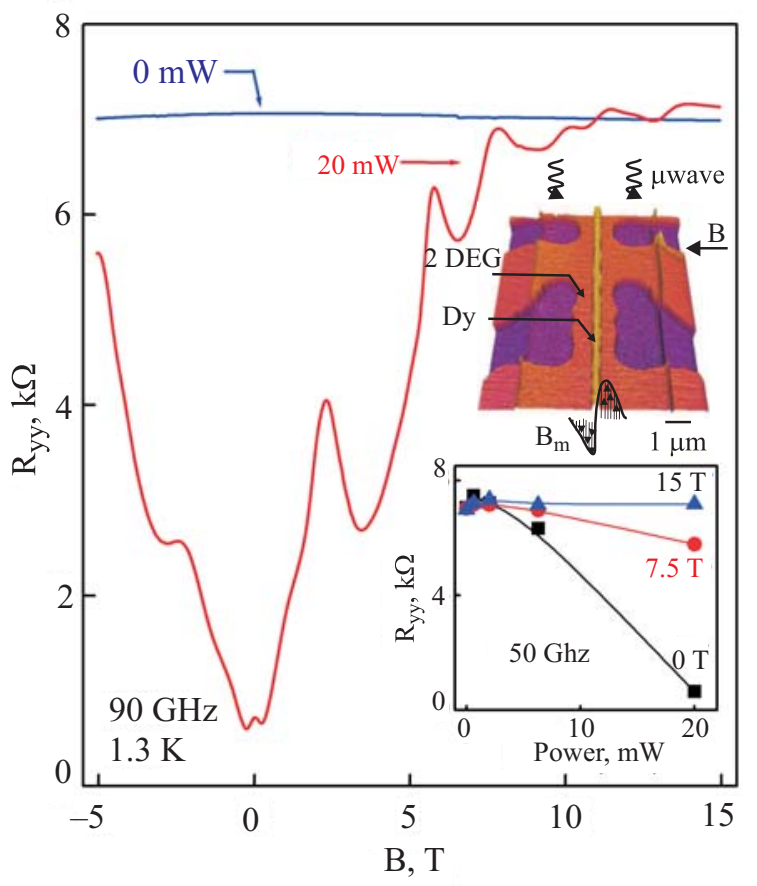

Fig. 1. Microwave induced forward scattering. Main panel: Resistance of a MCQW (200 nm wide, $24 \mu \mathrm{m}$ long) with and without microwave irradiation. The resistance at $B=0$ drops by over an order of magnitude when the microwave power is increased from 0 to $20 \mathrm{~mW}$. Top inset: A MCQW forms in a 2DEG confined laterally by the stray magnetic field $B_{m}(x)$ emanating from a microscopic bar magnet. The external magnetic field $B$ is applied in the plane to increase the perpendicular magnetization which in turn increases the depth of the magnetic quantum well. The photo-resistance is measured via four-terminal phase-locked detection under microwave irradiation. Bottom inset: Dependence of the photo-resistance on microwave power at $B=0$, 7.5 and $15 \mathrm{~T}$. The 0 and $7.5 \mathrm{~T}$ traces show a linear resistance decrease. The $15 \mathrm{~T}$ curve is essentially flat - only a small increase in resistance due to microwave heating is detectable. effects: the most notable features being two series of resistance oscillations and the recovery of the resistance level prior to irradiation when $B>9 \mathrm{~T}$. The resistance dependence on microwave power is shown in the inset to Fig. 1. To first approximation, the resistance decreases linearly for $B=0$ and $7.5 \mathrm{~T}$ whereas at $B=15 \mathrm{~T}$, the curve is independent of microwave power.

Figure 2, $a$ displays the evolution of the magnetoresistance at frequencies between $f=75$ and $110 \mathrm{GHz}$. The observed structure is an effect of the spatially varying magnetic field $B_{m}(x)$. Firstly, because the amplitude of peaks depends on the direction of the field sweep, a behavior associated with magnetization reversal in the micro-bar, see inset to Fig. 2,a. Secondly, one verifies that
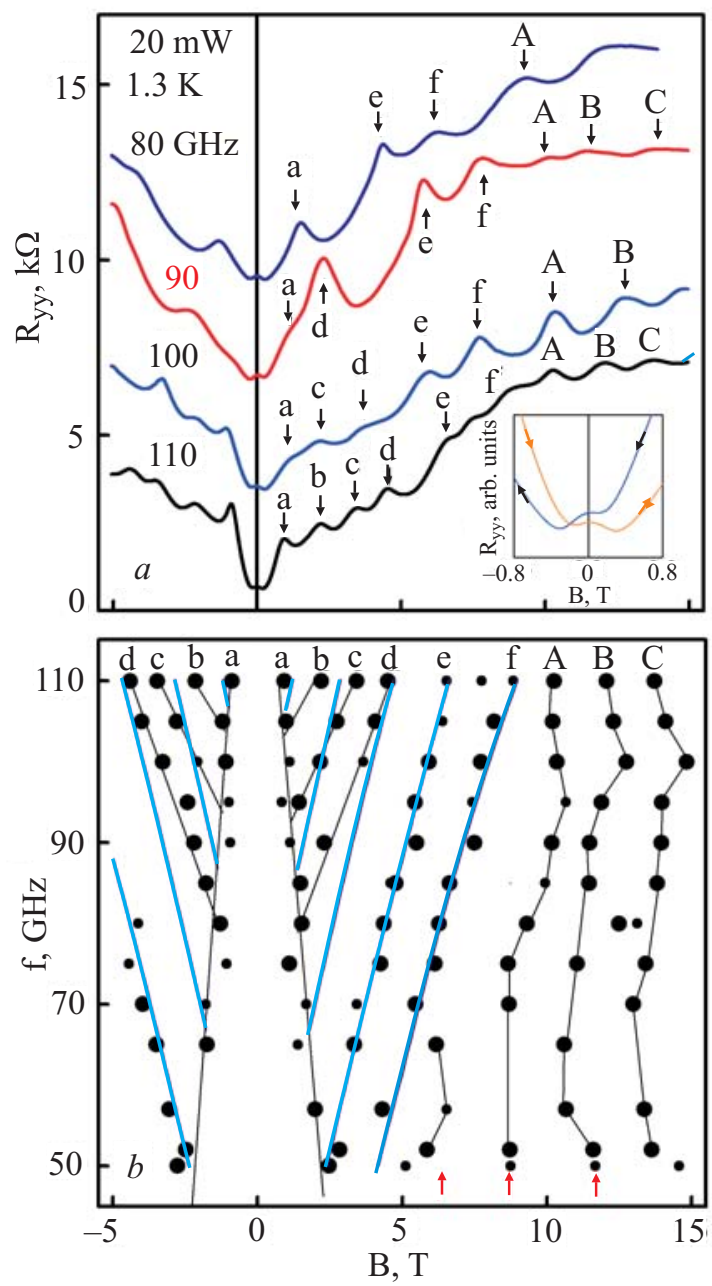

Fig. 2. Frequency dependence. (a) The longitudinal resistance displays two series of oscillations. The low-field series (a,b,c,d...) has peaks which move linearly with $f$. The peaks in the high-field series (A,B,C...) fluctuate about an average value which is independent of frequency. Curves are vertically offset by $3 \mathrm{k} \Omega$. Inset: magnetization reversal of dysprosium as evidenced by the longitudinal magnetoresistance. (b) Fan chart of peak positions. The dot size indicates the amplitude of experimental peaks. The blue lines (color online) are the theoretical fan fitted to the low-field series. The arrows indicate the theoretical peak positions of the high-field series (see text). 
bare Hall bars exhibit no magnetoresistance under similar experimental conditions [26]. The fan diagram in Fig. 2,b maps the position of individual peaks in the $f-B$ plane. One distinguishes two series of oscillations. The lowmagnetic field series has peaks labelled $(\mathrm{a}-\mathrm{f})$ whose position vary linearly with $f$. Peak (a) moves to lower magnetic field whereas peaks $(b-f)$ move to higher magnetic field. Peaks (A, B, C) are distinguished from the first series by being independent of microwave frequency. We shall see that, when $B>9 \mathrm{~T}$, the transverse magnetization of the micro-bar is within $4 \%$ of being saturated. As a result, tiny fluctuations in the magnetization easily produce very large variations in the $B$-values of peaks (A, B, C) as observed in Fig. 2, $b$.

The modulation field $B_{m}(x)$ induces a magnetic potential $V\left(x, k_{y}\right) \equiv \hbar^{2}\left[k_{y}+(e / \hbar) A_{y}(x)\right]^{2} / 2 m^{*}$ which depends on two parameters: the longitudinal momentum $k_{y}$ and the potential vector $A_{y}(x)=\int_{0}^{x} d \chi B_{m}(\chi)$ where $m^{*}$ is the electron effective mass. At high $B$, the stripe magnetization is saturated perpendicular to the stripe $\left(M_{\perp}=M_{\text {sat }}\right)$ which allows $B_{m}(x)$ to be calculated exactly as a function of the known dimensions of the stripe and the saturation magnetization of dysprosium $\left(\mu_{0} M_{\text {sat }}=3.67 \mathrm{~T}\right)$. The corresponding magnetic potential is shown in Fig. 3,a. One notable feature is the quantum well at the centre of the channel which defines the MCQW. This quantum well is deep enough $(25 \mathrm{meV})$ to confine the Fermi sea $(14.3 \mathrm{meV})$ and sufficiently narrow $(\sim 200 \mathrm{~nm})$ to support quantized energy levels separated by $\sim 0.5 \mathrm{meV}$, corresponding to $\sim 5 k_{B} T$ where $k_{B}$ is Boltzmann's constant. The energy subbands of the MCQW are plotted in Fig. 3, $b$ after numerically solving Schrödinger's equation in potential $V$. Looking at this diagram, one immediately sees that the effect of the magnetic potential is to segregate edge states carrying opposite currents to different regions of space. However, unlike in the quantum Hall effect where $N$ skipping orbits follow opposite edges, we have here $2 N$ magnetic edge states at the centre which propagate in the negative $y$ direction and $N$ free electron states at each edge of the channel which propagate along $y>0$, see the inset to Fig. $3, b$.

Our experiment suggests the formation of a Luttinger liquid in the MCQW. Firstly the pinning of a Luttinger liquid by impurities explains the anomalously large resistance of the wire in the absence of irradiation. Ogata and Fukuyama [27] have calculated the conductance of the dirty $\operatorname{LL}(G)$ relative to the conductance of noninteracting electrons $\left(G_{0}\right)$ as a function of the channel length $(L)$, the mean free path $(I)$, the Fermi temperature $\left(T_{F}\right)$ and $T_{L} \equiv \hbar v_{F} / k_{B} L$. Our MCQW has $L / l=6, T / T_{F}=8 \cdot 10^{-3}$ and $T_{L}=90 \mathrm{mK}$ for which the theory gives $G / G_{0} \approx 0.05$. The experimental ratio taken from Fig. 1 is $G(20 \mathrm{~mW}) / G(0)=0.08$. The good agreement between theory and experiment leads us to surmise that microwave-excited electron-hole pairs introduce attractive interactions in the Luttinger chain. These interactions switch
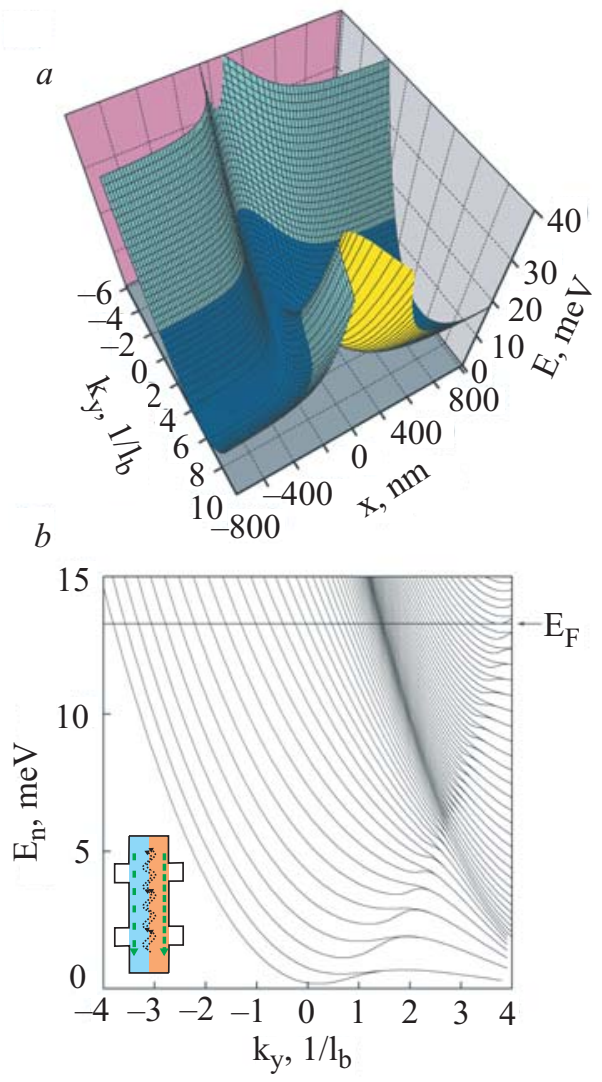

Fig. 3. Energy subbands of a magnetically confined quantum wire. (a) Magnetic potential $V\left(x, k_{y}\right)$ induced by the stray magnetic field $B_{m}(x)$ when the bar magnet is magnetized to full strength in the perpendicular direction: $\mu_{0} M_{\perp}=\mu_{0} M_{\text {sat }}=3.67 \mathrm{~T}$. The MCQW forms in the potential well at the centre of the channel. The Fermi sea is shown in dark. (b) Quantum energy subbands $E_{n}\left(k_{y}\right)$ supported by potential $V\left(x, k_{y}\right)$. Magnetic edge states bound to the MCQW - are found at the left of the shadowed parabola. To the right, the bonding/antibonding pairs are virtual subbands lying above the MCQW potential barrier. Further to the right, the group velocity changes sign and follows the free electron dispersion curve. Free electron edge states form at the edges of the Hall bar where $B_{m}$ vanishes. The length scale is $l_{b} \equiv \sqrt{\hbar / e}=25.7$ $\mathrm{nm}$. Inset: edge states in magnetically modulated Hall bar.

on forward scattering at pinning sites thus restoring perfect transmission as predicted by Kane and Fisher [2]. If the microwave heating of the electrons is inhibited as a result of a perturbation applied to the system, forward scattering will be switched off and the resistance will return to its level in the absence of irradiation. This is precisely what happens in a high magnetic field. Increasing the magnetic field increases the energy gaps between the confined electron states in the MCQW to the point where they become too large to absorb a microwave photon. The quantitative analysis done below sets the absorption edge at $8 \mathrm{~T}$ at $90 \mathrm{GHz}$ which corresponds well to the observed onset of the resistance plateau in Fig. 1.

Forward scattering reveals itself in more dramatic fashion in the magnetic field dependence of the resistance which we now describe. MCQWs have chiral edge states 
which by definition circulate in only one direction. This has the consequence that charge density excitations circulating along parallel LL channels will interfere in the fashion of a Mach-Zehnder interferometer if the channel paths are made to split and recombine at locations where the LLs interact. Such interferometer is shown in Fig. 3,a. Two weak links pin the LLs at $y$ and $y+d$. In the absence of microwaves, these pinning sites block transmission and cause charge accumulation as reported above. However in the presence of a microwave field tuned at the frequency of the inter-mode electron transition, electric dipoles allow a finite tunnelling probability through the pinning sites $[17,18]$. Two hybridized charge density waves propagate between $y$ and $y+d$. These have velocity given by

$$
s_{ \pm}=\frac{v_{l F}+v_{u F}}{2}+\frac{V_{0}}{\pi \hbar} \pm \frac{v_{l F}-v_{u F}}{2} \sqrt{1+K^{2}},
$$

where $v_{l F}$ and $v_{u F}$ are the Fermi velocities of the lower and the upper channel, $V_{0} \cong e^{2} / 4 \pi \varepsilon$ is the Coulomb interaction and $K=V_{0} / \hbar\left(v_{l F}-v_{u F}\right)$ is the effective coupling strength between channels [17]. We have noted $\varepsilon$ the dielectric constant of GaAs and $e$ the electron charge. When $B_{m}$ is maximum, one obtains $K=18$. Since $K$ will invariably increase for smaller $B_{m}$, the MCQW belongs to the strong coupling regime: $K \gg 1$. In which case, the speed of hybridized charge density waves is independent of $\left(v_{l F}-v_{u F}\right)$ hence independent of the magnetic field. These modes decay as $d^{-\lambda}$ where $\lambda_{ \pm} \equiv 1 \pm K / \sqrt{1+K^{2}}$. The mode $\lambda$ clearly dominates and is retained for fitting the peak positions. Theory predicts that this mode will absorb microwave power according to a $\sin \Phi$ function [17] where $\Phi$ is the phase factor given in the left hand side of Eq. (2). Maxima of power absorption occur when

$$
\left(k_{l F}-k_{u F}-\frac{2 \pi f}{s_{+}}\right) d+\frac{\pi}{2} \frac{K}{\sqrt{1+K^{2}}}=\frac{\pi}{2}+2 n \pi
$$
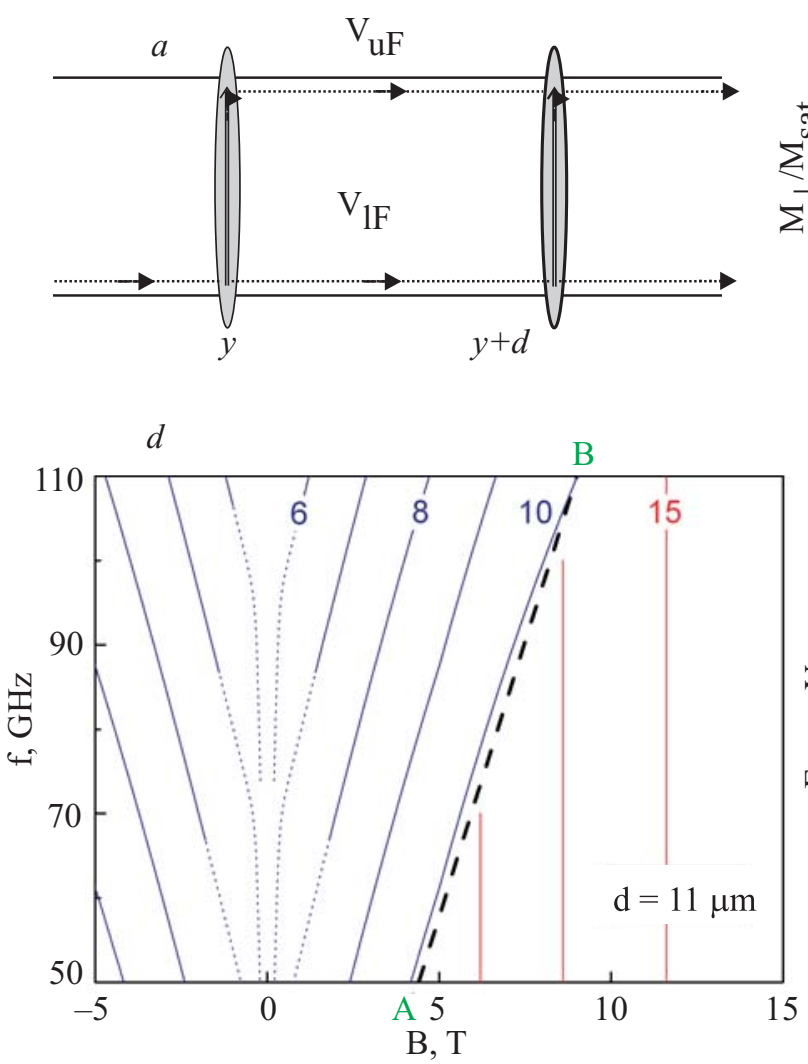
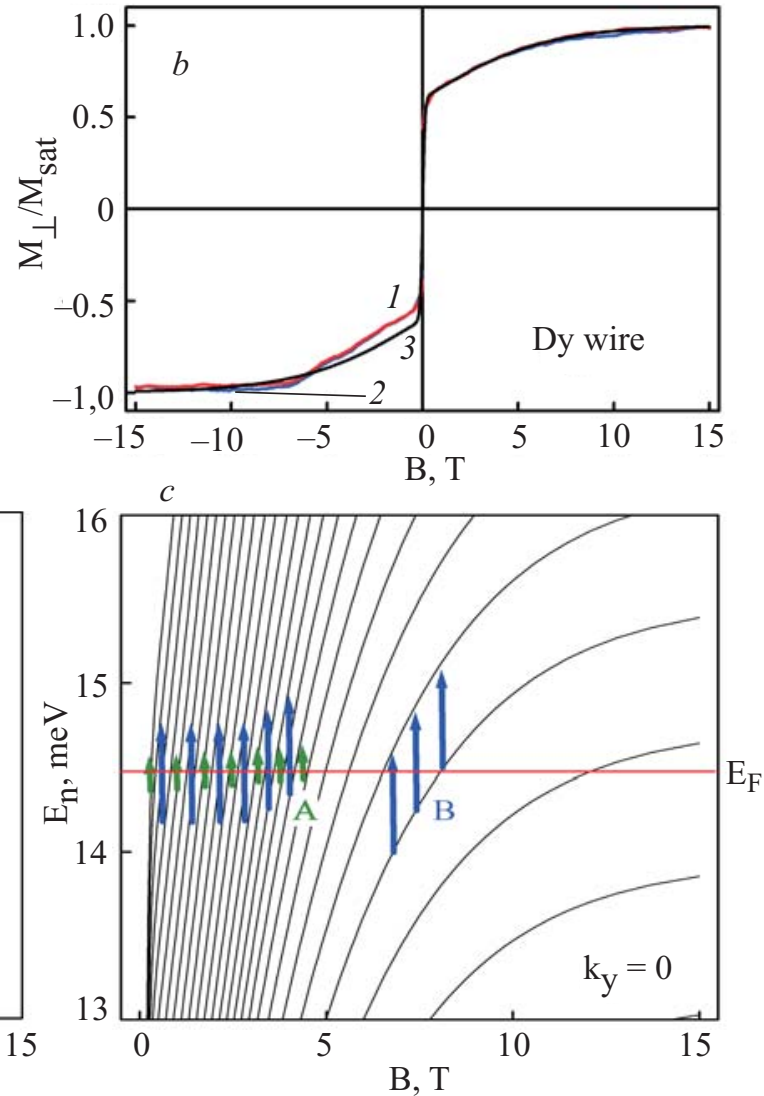

Fig. 4. Quantum interferences of chiral Luttinger channels in MCQW. (a) Resonant microwave absorption couples two Luttinger channels and enhances forward scattering at weak links (e.g., impurities) located at $y$ and $y+d$. Electron transmission through the interferometer depends on the difference in Fermi wavevectors and Fermi velocities of the lower and upper channels. (b) Transverse magnetization curve of a dysprosium bar - the up/down sweeps correspond to the curve $1 /$ curve 2 . Theoretical fit: $M / M_{\text {sat }}=0.6 \tanh (8 B)+0.4 \tanh (0.16 B)$ (curve 3). (c) Quantum energy levels $E_{n}\left(k_{y}=0, B\right)$ calculated as a function of the applied magnetic field using the empirical magnetization curve. Long (short) arrows show the electron transitions allowed at $110 \mathrm{GHz}(50 \mathrm{GHz})$. B (A) is the upper limit of microwave absorption at $110 \mathrm{GHz}(50 \mathrm{GHz})$. (d) The theoretical fan chart shows the microwave absorption frequencies of the Luttinger liquid as a function of the magnetic field. The absorption maxima are labelled $n=6,7,8,9,10$ (blue curves) and $n=13,14,15$ (red curves) (color online) as explained in the text. The dotted line (A-B) is the absorption edge. The distance $d=11 \mu \mathrm{m}$ is the only adjustment parameter in the theory. 
where $n$ is an integer and $k_{l F}-k_{u F}$ is the difference of Fermi wavevector of the lower and upper channels. The $k_{l F}-k_{u F}$ term carries the dependence on $B$. It thus becomes clear that Eq. (2) describes a fan diagram in the $f-B$ plane that has parallel branches, each indexed by $n$.

A quantitative fit of the experimental data requires knowing the magnetization curve $M_{\perp}=F(B)$. Figure $4, b$ shows the magnetization curve measured by Hall magnetometry together with the function used to interpolate it. The latter is used to calculate the $B$-dependence of energy levels, shown in Fig. 4,c, and the $B$-dependence of $k_{l F}-k_{u F}$. Inserting this into Eq. (2) gives the slope of every branch in the fan without any adjustment parameter. In contrast, the horizontal spacing between two consecutive branches, $n$ and $n+1$, depends on distance $d$ which we can extract from a fit to the data. The best fit to Fig. $2, b$ gives $d=(11 \pm 0.5) \mu \mathrm{m}$ (blue lines). We thus conclude that Coulomb interactions extend the range of quantum coherence to a distance at least 3 times the electron mean free path. The magnetic edge channel picture also explains why the fan only appears at low $B$. The energy gaps between MCQW subbands increase with $B$ up to a point where interchannel transitions are forbidden by energy conservation. Figure $4, c$ predicts the absorption edge to occur at $8.0 \mathrm{~T}(4.5 \mathrm{~T})$ at $110 \mathrm{GHz}(50 \mathrm{GHz})$ which fits nicely with the upper boundary of the experimental fan in Fig. 2,b.

We finally address the origin of the high-magnetic field series. This series is clearly nonresonant (as independent of $f$ ) and may be ascribed to charge density waves excited indirectly, perhaps in the contacts. Setting $f=0$ in Eq. (2) allows to calculate the maxima of microwave absorption as a function of $n$. The resulting theoretical positions are indicated by the arrows in Fig. 2,b which are in good agreement with the data. We summarize our findings by mapping the theoretical peak positions in Fig. 4,d. These replicate the major features of the experiment. Our results demonstrate the enhancement of quantum coherence by Coulomb interactions and incidentally the quantization of energy in a spatially varying magnetic field which is increasingly relevant to the development of carbon electronics [28,29].

\section{Acknowledgments}

We thank V.I. Fal'ko for stimulating discussions. The support of the EPSRC(UK) EP/E002390 and the Transnational Access - Specific Support Action Program Contract RITA-CT-2003-505474 of the European Commission is gratefully acknowledged.

1. For a review see: A.M. Chang, Rev. Mod. Phys. 75, 1449 (2003).

2. C.L. Kane and M.P.A. Fisher, Phys. Rev. Lett. 68, 1220 (1992).

3. C.L. Kane and M.P.A. Fisher, Phys. Rev. B46, 15233 (1992).
4. L.I. Glazman, I.M. Ruzin, and B.I. Shklovskii, Phys. Rev. B45, 8454 (1992).

5. M.D. Bockrath, D.H. Cobden, J. Lu, A.G. Rinzler, R.E. Smalley, L. Balents, and P.L. McEuen, Nature 397, 598 (1999).

6. O.M. Auslaender, A. Yacoby, R. de Picciotto, K.W. Baldwin, L.N. Pfeiffer, and K.W. West, Phys. Rev. Lett. 84, 1764 (2000).

7. B. Gao, A. Komnik, R. Egger, D.C. Glattli, and A. Bachtold, Phys. Rev. Lett. 92, 216804 (2004).

8. O.M. Auslaender, A. Yacoby, R. de Picciotto, K.W. Baldwin, L.N. Pfeiffer, and K.W. West, Science 295, 825 (2002).

9. M. Grayson, D.C. Tsui, L.N. Pfeiffer, K.W. West, and A.M. Chang, Phys. Rev. Lett. 86, 2645 (2001).

10. A. Yacoby, H.L. Störmer, N.S. Wingreen, L.N. Pfeiffer, K.W. Baldwin, and K.W. West, Phys. Rev. Lett. 77, 4612 (1996)

11. D. Loss, Phys. Rev. Lett. 69, 343 (1992).

12. K. Terabe, T. Hasegawa, T. Nakayama, and M. Aono, Nature 433, 47 (2005).

13. H. Onishi, Y. Kondo, and K. Takayanagi, Nature 395, 780 (1998).

14. J.I. Pascual, J. Mendez, J. Gomez-Herrero, A.M. Baro, N. Garcia, U. Landman, W. Luedtke, E.N. Bogachek, and H.P. Cheng, Science 267, 1793 (1995).

15. E. Slot, M.A. Holst, H.S.J. van der Zant, and S.V. Zaitsev-Zotov, Phys. Rev. Lett. 93, 176602 (2004).

16. K.A. Matveev and L.I. Glazman, Phys. Rev. Lett. 70, 990 (1993).

17. L.Y. Gorelik, I.V. Krive, S.I. Kulinich, R.I. Shekhter, and M. Jonson, Europhys. Lett. 57, 409 (2002).

18. I.V. Krive, S.I. Kulinich, L.Y. Gorelik, R.I. Shekhter, and M. Jonson, Phys. Rev. B64, 045114 (2001).

19. I.V. Kukushkin, M.Yu. Akimov, J.H. Smet, S.A. Mikhailov, K. von Klitzing, I.L. Aleiner, and V.I. Fal'ko, Phys. Rev. Lett. 92, 236803 (2004).

20. M. Bayer, P. Hawrylak, K. Hinzer, S. Fafard, M. Korkusinski, Z.R. Wasilewski, O. Stern, and A. Forchel, Science 291, 451 (2001).

21. D.P. DiVincenzo, Science 270, 255 (1995).

22. A. Vishwanath and T. Senthill, Phys. Rev. B63, 014506 (2001).

23. S.P. Strong, D.G. Clarke, and P.W. Anderson, Phys. Rev. Lett. 73, 1007 (1994).

24. X.G. Wen, Phys. Rev. B41, 12838 (1990).

25. D.N. Lawton, A. Nogaret, S.J. Bending, D.K. Maude, J.C. Portal, and M. Henini, Phys. Rev. B64, 033312 (2001).

26. C.L. Yang, R.R. Du, L.N. Pfeiffer, and K.W. West, Phys. Rev. B74, 045315 (2006).

27. M. Ogata and H. Fukuyama, Phys. Rev. Lett. 73, 468 (1994).

28. A. De Martino, L. Dell'Anna, and R. Egger, Phys. Rev. Lett. 98, 066802 (2007).

29. J.R. Shi, F.M. Peeters, K.W. Edmonds, and B.L. Gallagher, Phys. Rev. B66, 035328 (2002). 